\title{
Erratum to: Real-Time Progressive Hyperspectral Image Processing
}

\author{
Chein-I Chang
}

\section{Erratum to:}

C.-I Chang, Real-Time Progressive Hyperspectral Image Processing, DOI 10.1007/978-1-4419-6187-7

The volume was originally published without the About the Author information that has now been added to the frontmatter pages.

The updated original online version for this book frontmatter can be found at DOI 10.1007/978-1-4419-6187-7

Chein-I Chang $(\square)$

Baltimore, MD, USA

e-mail: cchang@umbc.edu 